\title{
O conhecimento dos profissionais de saúde sobre vacinação de pessoas vivendo com HIV - uma revisão integrativa
}

\author{
Health professionals' knowledge about vaccination of people living with HIV - an integrative review \\ El conocimiento de los profesionales de la salud sobre la vacunación de personas que viven con VIH - \\ revisión integradora
}

\author{
Larissa Gerin ${ }^{1,2}$ (1) \\ Marcela Antonini² (1) \\ Karen da Silva Santos ${ }^{3,4}$ (i) \\ Elucir $\mathrm{Gir}^{5}$ (1) \\ Renata Karina Reis ${ }^{5}$ (])
}

1. Secretaria Municipal de Saúde de Ribeirão

Preto. Ribeirão Preto, SP, Brasil.

\section{Universidade de São Paulo, Escola de}

Enfermagem de Ribeirão Preto, Programa

de Pós-Graduação em Enfermagem

Fundamental. Ribeirão Preto, SP, Brasil.

3. Universidade de São Paulo, Escola de Enfermagem de Ribeirão Preto, Programa de Pós-Graduação em Enfermagem em Saúde Pública. Ribeirão Preto, SP Brasil.

4. Universidade de Limoges, Laboratório de Education et Diversité en Espaces

Francophones, Limoges, França.

5. Universidade de São Paulo, Escola de Enfermagem de Ribeirão Preto, Departamento de Enfermagem Geral e Especializada. Ribeirão Preto, SP, Brasil.
Autor correspondente:

Larissa Gerin.

E-mail: larissagerin@yahoo.com.br

Recebido em 17/06/2021.

Aprovado em 26/08/2021.

\section{Resumo}

Objetivo: Identificar evidências na literatura acerca do conhecimento dos profissionais de saúde sobre vacinação das pessoas vivendo com HIV. Método: Trata-se de uma revisão integrativa. Para a busca, foram utilizados os descritores: pessoal de saúde (health personnel), conhecimento (knowledge), vacinação (vaccination), HIV e seus sinônimos, sem utilização de filtros, nas bases de dados Pubmed, Biblioteca Virtual em Saúde, Web of Science, Cumulative Index to Nursing and Allied Health Literature, Embase, Scopus, Science Direct e Cochrane. Foram incluídos apenas artigos primários analisados por meio do aplicativo RAYYAN. Resultados: De 601 publicações iniciais, apenas cinco constituíram a amostra final, todas identificadas no Pubmed publicadas entre 2013 e 2018, sendo nenhum estudo brasileiro. A maioria das publicações estava relacionada a alguma vacina específica e não abordava todo o calendário vacinal. Conclusão e implicações para a prática: $O$ déficit de conhecimento dos profissionais de saúde, em relação às vacinas indicadas às pessoas vivendo com HIV, foi o principal aspecto identificado, resultando em insegurança dos profissionais. Há a necessidade de educação permanente das equipes multiprofissionais dos serviços especializados e da atenção primária visando diminuir as barreiras e aumentar a cobertura vacinal desta clientela.

Palavras-chave: Cobertura vacinal; Conhecimento; HIV; Profissionais de saúde; Vacinação.

\section{Abstract}

Objective: to identify evidence in the literature about the health professionals' knowledge concerning vaccination of people living with HIV. Method: This is an integrative review. In this research, the descriptors used were the following: health personnel, knowledge, vaccination, HIV and its synonyms, without the use of filters, in the Pubmed, Virtual Health Library, Web of Science, Cumulative Index to Nursing and Allied Health Literature, Embase, Scopus, Science Direct and Cochrane databases. Only primary articles analyzed using the RAYYAN application were included. Results: Out of 601 initial publications, only five comprised the final sample, all identified in Pubmed and published between 2013 and 2018, with no Brazilian studies. Most of the publications were related to a specific vaccine and did not address the entire vaccination schedule. Conclusion and implications for the practice: The health professionals' lack of knowledge in relation to vaccines indicated to people living with HIV was the main aspect identified, resulting in insecurity among the professionals. There is a need for permanent education of the multidisciplinary teams of specialized services and primary care in order to reduce barriers and to increase vaccination coverage for this clientele.

Keywords: Vaccination coverage; Knowledge; HIV; Health professionals; Vaccination.

\section{Resumen}

Objetivo: Identificar evidencias en la literatura acerca del conocimiento de los profesionales de la salud sobre la vacunación de personas que viven con VIH. Método: Revisión integradora. Para la búsqueda, se utilizaron los descriptores: personal de salud (health personnel), conocimiento (knowledge), vacunación (vaccination), VIH y sus sinónimos, sin el uso de filtros, en las bases de datos Pubmed, Biblioteca Virtual en Salud, Web of Science, Cumulative Index to Nursing and Allied Health Literature, Embase, Scopus, Science Direct y Cochrane. Solo se incluyeron los artículos primarios analizados mediante la aplicación RAYYAN. Resultados: De 601 publicaciones iniciales, solo cinco constituyeron la muestra final, todas identificadas en Pubmed y publicadas entre 2013 y 2018, sin ningún estudio brasileño. La mayoría de las publicaciones estaban relacionadas con una vacuna específica y no abordaban todo el calendario de vacunación. Conclusión e implicaciones para la práctica: El desconocimiento de los profesionales de la salud en relación a las vacunas indicadas para personas que viven con VIH fue el principal aspecto identificado, lo que generó inseguridad entre los profesionales. Se advierte la necesidad de disponer la educación permanente de los equipos multiprofesionales de servicios especializados y de atención primaria para reducir barreras y aumentar las coberturas de vacunación de esta clientela.

Palabras clave: Cobertura de vacunación; Conocimiento; VIH; Profesionales de la salud; Vacunación. 


\section{INTRODUÇÃO}

Nos últimos anos, ocorreram muitas mudanças em relação às características epidemiológicas da infecção pelo vírus da imunodeficiência humana (HIV). De acordo com a Joint United Nations Program on AIDS/HIV (UNAIDS) cerca de 38 milhões de pessoas vivem com HIV (PVHIV) no mundo e 690.000 indivíduos morreram em consequência da aids até $2020^{1}$.

No Brasil, a aids é de notificação compulsória desde 1986, e a infecção pelo HIV desde 2014. Quanto ao número de casos, o que se observa é uma diminuição do percentual dos casos de aids com uma redução de $18,7 \%$ na taxa de detecção a partir de 2012 e de $28,1 \%$ na taxa de mortalidade de 2014 a 2019. Por outro lado, os números de casos da infecção pelo HIV seguem aumentando no país, sendo que em 2019 foram diagnosticados 41.909 novos casos ${ }^{2}$.

Nos últimos anos, o modelo de atenção a PVHIV no Brasil tem sofrido mudanças com a participação crescente dos serviços de atenção básica no desenvolvimento de ações de promoção, prevenção, diagnóstico e acompanhamento dessa clientela na atenção primária à saúde $(\mathrm{APS})^{3}$.

A redução no número de casos de aids e óbitos no Brasil está diretamente relacionada ao tratamento proposto pelo Ministério da Saúde com a "cascata de cuidado contínuo ao HIV", que incentiva o diagnóstico precoce, a vinculação e retenção do indivíduo ao serviço de saúde e o início precoce da Terapia Antirretroviral (TARV) com o objetivo de suprimir a carga viral ${ }^{4}$.

Com os avanços observados no cuidado às PVHIV, a infecção se tornou uma condição crônica, os indivíduos têm alcançado maior sobrevida e melhor qualidade de vida e, consequentemente, se expõem mais ao risco de adquirir outras doenças infecciosas, incluindo, as imunopreveníveis. Neste contexto, destaca-se a importância das ações de promoção à saúde e prevenção de doenças junto a esta população em todas as esferas das redes atenção à saúde, o que inclui as ações de imunização ${ }^{5}$.

O quadro da infecção pelo HIV é bastante heterogêneo, podendo variar desde a imunocompetência até uma grave imunodeficiência, que coloca as PVHIV em risco aumentado para muitas infecções virais, bacterianas e fúngicas ${ }^{6}$. Assim, é primordial compreender o grau de imunodepressão do indivíduo para a indicação de vacinas. A vacinação de indivíduos com alto grau de imunodepressão, por meio de vacinas com agentes vivos atenuados, aumenta a possibilidade de eventos adversos e caso sejam vacinados com vacinas inativadas a resposta pode ser insuficiente ${ }^{7}$.

Para as PVHIV existem recomendações específicas de vacinação em função deste grupo apresentar maior risco de morbimortalidade por doenças que podem ser prevenidas por vacinas $^{8,9}$. Por ser um grupo bastante específico e heterogêneo em que a resposta vacinal depende do grau de imunodepressão e que pode desenvolver eventos adversos a partir da vacinação inadvertida em casos de alto grau de imunodepressão, a complexidade dos aspectos envolvidos na vacinação de PVHIV gera diversas dúvidas nos profissionais de saúde ${ }^{7}$.
Apesar do aumento do risco de infecções e da disponibilidade de número cada vez maior de vacinas, as taxas de vacinação em PVHIV permanecem mais baixas do que na população em geral ${ }^{6,9}$.

É recomendado que a avaliação da situação de saúde (sinais e sintomas clínicos), do histórico de doenças prévias e do histórico vacinal do indivíduo que recebe o diagnóstico da infecção pelo HIV seja realizada durante o primeiro atendimento no serviço onde realiza o acompanhamento. A partir da avaliação dos exames de primeira consulta (contagem de células T CD4, carga viral, sorologias anti-HBs e anti-HAV IgG) é recomendada a atualização do esquema vacinal, sendo que esta recomendação pode ser realizada por qualquer um dos profissionais de saúde das equipes multiprofissionais que acompanham o indivíduo, especialistas ou profissionais da APS $^{4,9}$.

Profissionais de saúde devidamente capacitados para os esquemas de vacinas destinados às PVHIV podem contribuir na melhora da cobertura vacinal nesta clientela, avaliando o histórico vacinal dos indivíduos e indicando as vacinas apropriadas de acordo com os protocolos estabelecidos, para que assim contribuam para a redução na morbimortalidade por infecções evitáveis por vacinas.

A atualização dos esquemas de vacinação das PVHIV deve ocorrer antes que a imunodepressão se instale; caso já esteja instalada cabe ao profissional de saúde verificar a possibilidade de adiar a vacinação até que o sistema imunológico seja restaurado. Além disso, é importante que seja avaliada e, caso necessário seja atualizada, a situação vacinal dos profissionais de saúde envolvidos no atendimento de PVHIV e dos contatos domiciliares desses pacientes, visando a prevenção de doenças evitáveis por vacina, principalmente para aqueles com contraindicação de receber algum imunobiológico. ${ }^{7,9}$

Entretanto, pouco se sabe sobre o conhecimento dos profissionais de saúde em relação a este tema, por este motivo e considerando a problemática enquanto uma realidade de saúde pública, este trabalho tem o objetivo identificar na literatura os aspectos envolvidos no conhecimento dos profissionais de saúde em relação a vacinação das PVHIV.

\section{MÉTODO:}

O presente estudo é uma revisão integrativa (RI), instrumento da prática baseada em evidência, realizado de forma sistemática e rigorosa, que permite a inclusão de diversos métodos de pesquisa, experimentais e não experimentais, para uma ampla compreensão do fenômeno estudado ${ }^{10-12}$.

Este estudo foi desenvolvido a partir das seguintes etapas: elaboração da questão de pesquisa, estabelecimento dos critérios de elegibilidade, busca na literatura, definição das informações a serem extraídas dos estudos selecionados, coleta de dados, análise crítica das publicações selecionadas, interpretação dos resultados e apresentação/síntese do conhecimento ${ }^{11,12}$.

A questão de pesquisa foi construída a partir do acrônimo PICo, sendo a "população" $(P)$ os profissionais de saúde, o fenômeno de "interesse" (I) o conhecimento e o "contexto" (Co) que foi a vacinação das pessoas vivendo com HIV ${ }^{13}$. Essa 
questão, portanto, foi: "qual é o conhecimento dos profissionais de saúde em relação à vacinação das PVHIV?".

A busca dos estudos ocorreu nas bases de dados Pubmed, Biblioteca Virtual em Saúde (BVS), Web of Science, Cumulative Index to Nursing and Allied Health Literature (CINAHL), Embase, Scopus (Elsevier), Science Direct e Cochrane durante o mês de dezembro de 2020. Foi realizada também a busca manual nas referências dos artigos selecionados para leitura na íntegra.

A estratégia de busca se constituiu por descritores e seus sinônimos identificados nos Descritores em Ciência da Saúde (DeCS) e seus equivalentes em inglês identificados no Medical Subject Headings (MeSH). Entre os descritores e seus sinônimos foi utilizado o operador booleano OR dentro dos conjuntos de termos da estratégia PICo, e para o cruzamento dos grupos de descritores foi utilizado o operador booleano AND (Quadro 1).

Os arquivos de busca de cada base de dados foram importados para o aplicativo RAYYAN onde foi realizada a avaliação de título e resumo, por dois revisores independentes, a partir dos critérios de elegibilidade, e um terceiro revisor resolveu as discordâncias ${ }^{14}$.

Foram incluídos artigos primários que apresentassem dados referentes ao conhecimento de profissionais de saúde em relação à vacinação de PVHIV, seja para todo o calendário vacinal ou para vacinas específicas com indicação para este público. Para os artigos selecionados foi realizada a extração das informações em uma planilha desenvolvida no Excel pelos autores a partir da adaptação de instrumento já validado com as seguintes variáveis: título do artigo, base de dados onde foi identificado, título do periódico, nome dos autores, país, idioma, ano de publicação, instituição sede do estudo, delineamento, objetivo, amostra, critérios de inclusão/exclusão, resultados, análise dos dados, conclusões, nível de evidência e avaliação do rigor metodológico ${ }^{10,12,15}$.

Para determinar o nível de evidência, foi utilizada a seguinte classificação: nível I - metanálise de ensaios clínicos randomizados e controlados, nível II - ensaios clínicos randomizados e controlados, nível III - ensaios clínicos sem aleatorização, nível IV - estudos de caso-controle e coorte, nível $\mathrm{V}$ - revisões sistemáticas, estudos descritivos e qualitativos, nível VI - opiniões de autoridades e/ ou parecer de comitês de especialistas. Para avaliar o rigor metodológico foi verificada a clareza na trajetória metodológica e a identificação de vieses ou limitações ${ }^{10,12,15,16}$.

Foram identificadas 601 publicações, sendo 155 registros duplicados e 436 foram excluídas após a análise dos critérios de elegibilidade. Ao buscar os estudos na íntegra foram excluídos dois trabalhos que não se tratavam de estudos primários, um deles consistia de estudo publicado em anais de trabalho apresentado em congresso e outro de um projeto inscrito em um protocolo de pesquisa. Na busca manual não foi identificada nenhuma publicação que atendesse aos critérios de elegibilidade.

Para a leitura na íntegra ficaram oito publicações, após a leitura foram excluídas três, sendo que duas delas abordavam a imunização das PVHIV, porém, não abordavam sobre o conhecimento dos profissionais a respeito do tema e outro estudo abordava o conhecimento em relação a vacina contra Herpes Zoster, porém, não estava relacionado ao público que vive com o HIV (Figura 1).

A análise crítica das publicações selecionadas e a síntese do conhecimento foram realizadas de forma descritiva a partir dos dados coletados na planilha de extração de dados. Através da análise temática, as variáveis foram descritas e posteriormente

Quadro 1. Estratégias de busca em português e inglês utilizadas nas bases de dados Biblioteca Virtual em Saúde e Pubmed. Ribeirão Preto, SP, Brasil, 2020.

\begin{tabular}{|c|c|}
\hline Base de dados & Estratégia de busca utilizada \\
\hline BVS & $\begin{array}{l}\text { ("pessoal de saúde" OR "prestadores de cuidados de saúde" OR "profissionais da saúde" OR "profissionais } \\
\text { de saúde" OR "profissional da saúde" OR "profissional de saúde" OR "trabalhador da saúde" OR } \\
\text { "trabalhador de saúde" OR "trabalhadores da saúde" OR "trabalhadores de saúde") AND (conhecimento } \\
\text { OR conhecer OR conhecimentos OR epistemologia OR "unidade de conhecimento" OR "unidade do } \\
\text { conhecimento" OR "unidades de conhecimento" OR "unidades do conhecimento") AND (vacinação OR } \\
\text { "imunização ativa") AND (HIV OR "vírus da AIDS" OR "vírus da imunodeficiência humana" OR "vírus de } \\
\text { imunodeficiência humana") }\end{array}$ \\
\hline Pubmed & $\begin{array}{l}\text { ("health personnel" OR "personnel, health" OR "health care providers" OR "health care provider" OR } \\
\text { "provider, health care" OR "healthcare providers" OR "healthcare provider" OR "provider, healthcare" } \\
\text { OR "healthcare workers" OR "healthcare worker" OR "health care professionals" OR "health care } \\
\text { professional" OR "professional, health care") AND (knowledge OR epistemology) AND (vaccination OR } \\
\text { "vaccinations" OR "immunization, active" OR "active immunization" OR "active immunizations" OR } \\
\text { "immunizations, active") AND (HIV OR "human immunodeficiency virus" OR "immunodeficiency virus, } \\
\text { human" OR "immunodeficiency viruses, human" OR "virus, human immunodeficiency" OR "viruses, } \\
\text { human immunodeficiency" OR "human immunodeficiency viruses" OR "AIDS virus" OR "AIDS viruses" } \\
\text { OR "virus, AIDS" OR "viruses, AIDS" OR "acquired immune deficiency syndrome virus" OR "acquired } \\
\text { immunodeficiency syndrome virus") }\end{array}$ \\
\hline
\end{tabular}

Fonte: Dados da Revisão, Ribeirão Preto/SP, 2021 


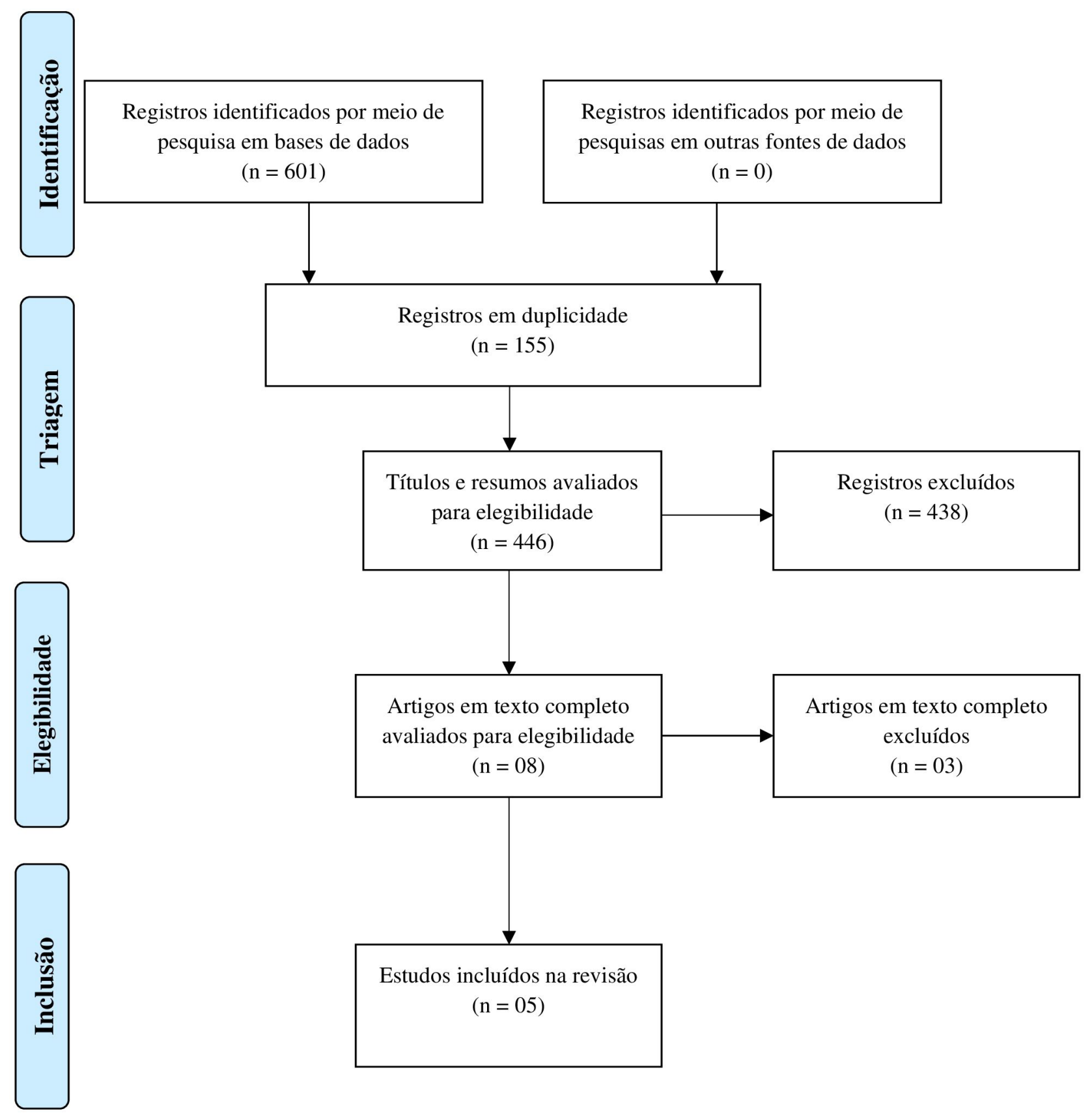

Figura 1. Fluxograma do processo de seleção dos estudos. ${ }^{17}$

Fonte: Ribeirão Preto, SP, Brasil, 2021

interpretadas buscando-se tópicos comuns entre os estudos que então foram divididos em duas categorias: estudos que avaliaram todo o esquema de vacinação indicado para as PVHIV e estudos que avaliaram apenas uma vacina indicada para as PVHIV ${ }^{18}$.

\section{RESULTADOS}

A amostra final se constituiu em cinco artigos, os anos de publicação foram um em 2013 (20,0\%), dois em 2016 (40,0\%), um em $2017(20,0 \%)$ e um em $2018(20,0 \%)$, todos (100\%) em inglês. Foram 3 trabalhos (60,0\%) realizados nos EUA, um (20,0\%) no Reino Unido e outro (20,0\%) na África do Sul (Tabela 1).

A instituição sede de dois estudos (40,0\%) foram universidades, um estudo (20,0\%) foi realizado por um Centro de Medicina do Viajante e dois são estudos multicêntricos (40,0\%).

Em relação à população do estudo, três estudos $(60,0 \%)$ tiveram como critério de inclusão que o profissional que respondesse ao questionário atendesse PVHIV em sua prática profissional, para outros dois estudos (40,0\%) este não foi um critério de inclusão de profissionais na pesquisa. Todos os artigos 
Tabela 1. Publicações selecionadas segundo ano de publicação, país de realização do estudo, título, periódico e autores. Ribeirão Preto, SP, Brasil, 2021.

\begin{tabular}{|c|c|c|c|c|c|}
\hline $\mathrm{N}$ & Ano & País & Título & Periódico & Autores \\
\hline 1 & 2013 & EUA & $\begin{array}{l}\text { Providers' lack of knowledge about herpes zoster } \\
\text { in HIV-infected patients is among barriers to herpes } \\
\text { zoster vaccination. }\end{array}$ & $\begin{array}{l}\text { International } \\
\text { Journal of } \\
\text { STD \& AIDS }\end{array}$ & $\begin{array}{l}\text { Aziz M, Kessler K, } \\
\text { Huhn G }\end{array}$ \\
\hline 2 & 2016 & Reino Unido & $\begin{array}{l}\text { Enquiries to the United Kingdom National Travel } \\
\text { Advice Line by healthcare professionals regarding } \\
\text { immunocompromised travellers. }\end{array}$ & $\begin{array}{l}\text { Journal } \\
\text { of Travel } \\
\text { Medicine }\end{array}$ & Allen JE, Patel D \\
\hline 3 & 2016 & EUA & $\begin{array}{l}\text { Knowledge of Vaccination Needs of HIV-Infected } \\
\text { Men Who Have Sex with Men in a National Sample } \\
\text { of "Gay Friendly" Health Care Providers. }\end{array}$ & $\begin{array}{l}\text { Public Health } \\
\text { Nursing }\end{array}$ & Blackwell CW \\
\hline 4 & 2017 & África do Sul & $\begin{array}{l}\text { Knowledge, attitudes and practices of South African } \\
\text { healthcare workers regarding the prevention } \\
\text { and treatment of influenza among HIV-infected } \\
\text { individuals. }\end{array}$ & PLOS ONE & $\begin{array}{l}\text { Duque J, Gaga S, } \\
\text { Clark D, Muller M, } \\
\text { Kuwane B, } \\
\text { Cohen C et al. }\end{array}$ \\
\hline 5 & 2018 & EUA & $\begin{array}{l}\text { HPV-Related Cancer Prevention and Control } \\
\text { Programs at Community-Based HIV/AIDS Service } \\
\text { Organizations: Implications for Future Engagement. }\end{array}$ & $\begin{array}{l}\text { Frontiers in } \\
\text { Oncology }\end{array}$ & $\begin{array}{l}\text { Wigfall LT, Bynum SA, } \\
\text { Brandt HM, } \\
\text { Sebastian N, Ory MG }\end{array}$ \\
\hline
\end{tabular}

Fonte: Dados da Revisão, Ribeirão Preto/SP, 2021

selecionados (100\%) foram classificados com nível de evidência V, pois são estudos descritivos, com abordagem quantitativa, não experimentais (estudos transversais) ${ }^{15}$. Todos os estudos (100\%) foram realizados com amostras selecionadas por conveniência.

Na primeira categoria de estudos, dois avaliaram o conhecimento geral em relação à vacinação do público imunodeprimido ou especificamente HIV, um deles relacionado à imunização do viajante imunodeprimido ${ }^{19} \mathrm{e} o$ outro em relação à imunização de homens que fazem sexo com homens (HSH) infectados pelo $\mathrm{HIV}^{20}$. Os estudos demonstraram que os profissionais de saúde que atendem PVHIV apresentam muitas dúvidas em relação às vacinas indicadas para este público e suas contraindicações, principalmente em relação às vacinas atenuadas. Os autores reforçam que as dúvidas entre os profissionais de saúde permanecem apesar dos países disponibilizarem protocolos de imunização estabelecidos (Tabela 2).

Ao avaliarem as ligações telefônicas de profissionais de saúde a um centro de saúde do viajante no Reino Unido para aconselhamento em relação a viajantes imunocomprometidos, verificaram que a maioria dos contatos eram relacionados a dúvidas sobre vacina, principalmente sobre vacinas atenuadas, apesar de muitos profissionais também apresentarem dúvidas em relação à indicação de vacinas inativadas. Ligações para aconselhamento em relação aos viajantes com HIV representou $11 \%$ do total, porém, destes, apenas $32 \%$ tinham conhecimento sobre a contagem de células T CD4 e apenas $27 \%$ sobre a carga viral. Neste estudo, apesar de uma amostra com tamanho adequado, a coleta de dados foi realizada de forma retrospectiva, o que limitou a análise dos dados pelo fato de faltar informações importantes (como o nível de imunodepressão dos pacientes) ${ }^{19}$.

Um outro estudo, realizado nos EUA, avaliou o conhecimento de profissionais de saúde de uma lista de profissionais "gay friendly" em relação às necessidades de vacina para populações de alto risco como a de homens que fazem sexo com homens (HSH) tanto soropositivos quanto soronegativos para o HIV. Do grupo de profissionais avaliados, apenas $11 \%$ identificaram corretamente as vacinas indicadas aos HSH soropositivos para o HIV. Os profissionais da atenção primária à saúde apresentaram maior habilidade para identificar corretamente as vacinas indicadas para este público, assim como aqueles que administram vacinas na sua prática, e aqueles com maior familiaridade com os protocolos de vacinação. A amostra do estudo foi pequena, não representativa da população, com um viés de motivação (a maioria dos participantes se identificaram como homossexuais) e proveniente de uma única fonte de dados (lista de profissionais inscritos na Associação Médica de Gays e Lésbicas), fatores estes que limitaram o estudo ${ }^{20}$.

$\mathrm{Na}$ segunda categoria, foram incluídos três estudos que avaliaram o conhecimento dos profissionais de saúde em relação a apenas uma das vacinas indicadas para as PVHIV. Um dos estudos avaliou nos EUA o conhecimento de profissionais de saúde que atendiam PVHIV em relação à incidência e gravidade do herpes zoster para verificar se este conhecimento e a prática dos profissionais com imunização contribuiria para recomendar a vacinação contra a doença. A maioria dos profissionais do estudo indicava para as PVHIV as vacinas do calendário vacinal do país, porém, desconhecia os princípios da vacinação contra 
Tabela 2. Publicações selecionadas segundo resultados e limitações ou vieses de acordo com a categoria temática em que foi inserido para análise. Ribeirão Preto, SP, Brasil, 2021.

\begin{tabular}{l} 
Nesultados \\
\hline Categoria 1 - Estudos que avaliaram todo o esquema de vacinação indicado para PVHIV \\
Os profissionais de saúde (PS) vacinaram rotineiramente seus pacientes na prática - \\
Influenza (91,2\%), Hepatite A (86,0\%), Hepatite B (92,4\%), Pneumo (87,2\%), tétano \\
(75\%). Mais de 75\% dos PS não vacinam seus pacientes com HIV contra varicela zoster. \\
Barreiras para a vacinação: segurança não comprovada da vacina, preocupaçães com \\
a eficácia, riscos de disseminação, problemas com seguro/reembolso, as diretrizes do \\
Infectious Diseases Society of America (IDSA) não recomendam a vacinação.
\end{tabular}

267 inquéritos (67\%) foram sobre vacina; 77 inquéritos (20\%) foram sobre vacinas e profilaxia da malária; 44 inquéritos (11\%) foram sobre a profilaxia da malária. Dos inquéritos relacionados à vacina (344),60\% estavam relacionados a vacinas vivas,

$323 \%$ a vacinas inativadas e $17 \%$ a ambas as vacinas. Do total de inquéritos, 44 (11\%) foram sobre viajantes com HIV. A contagem de linfócitos T CD4 era conhecida apenas para 14 viajantes (32\%) e a carga viral (CV) para 12 viajantes $(27 \%)$ - 10 indetectável. Foi identificado o uso de TARV para 22 viajantes - 21 (48\%) utilizavam e 1 (2,3\%) não utilizava.

\section{Categoria 2 - Estudos que avaliaram apenas uma vacina indicada para PVHIV}

Cerca de $11 \%$ identificaram corretamente as vacinas necessárias para HSH infectados pelo HIV. As variáveis estatisticamente significativas associadas ao maior conhecimento da necessidade de vacinação foram: maior familiaridade com o esquema de vacinação de adultos, especialização prática, administração de vacinas no local de trabalho.

70\% pensam que a vacinação do PS ajuda a proteger os pacientes contra influenza; a razão mais importante para se vacinar é proteger a sua família e os pacientes; $34 \%$ relataram ter recebido a vacina na última sazonalidade, $63 \%$ receberam alguma vez na vida (os médicos foram os profissionais menos propensos à vacinação). Variáveis

4 relacionadas a receber a vacina: acesso, vacina disponível sem custo e ter recebido treinamento de influenza. $94 \%$ dos profissionais recomendaram a vacina para seus pacientes. Variáveis relacionadas à maior probabilidade de recomendar a vacina: vacinação autorrelatada 2013/2014; disponibilidade da vacina durante a consulta, conhecer as diretrizes de vacinação e ter recebido treinamento sobre influenza.

$77 \%$ tinham ouvido falar da vacina contra o HPV, $52 \%$ achavam que a vacina era segura 5 e $43 \%$ achavam ser eficaz, $47 \%$ achavam que a vacina pode prevenir contra o câncer cervical. Todos os funcionários estavam dispostos a encorajar as mulheres e HSH para falar com seus provedores sobre a vacina contra o HPV.
Limitações ou Vieses

A taxa de resposta foi baixa e pode não ser representativa de todos os PS que atendem PVHIV, dependia do autorrelato (as práticas e comportamentos não foram observados).

Estudo retrospectivo; dependeu do registro do enfermeiro/ aconselhador, faltou informações clínicas (não era o objetivo principal).

Fonte: Dados da Revisão, Ribeirão Preto/SP, 2021

o herpes zoster ou não estava seguro na indicação da vacina. Um fator limitador do estudo é o tamanho da amostra, que não é representativa em relação a todos os profissionais de saúde e as respostas foram baseadas em autorrelato de profissionais inscritos em um programa de educação continuada em HIV ${ }^{21}$.

Um outro estudo avaliou junto aos profissionais de saúde de hospitais públicos, clínicas e centros de saúde de cinco províncias sul-africanas o conhecimento, atitudes e práticas relacionadas à prevenção e ao tratamento da gripe, especialmente para PVHIV. Dos profissionais que responderam o instrumento utilizado na pesquisa, $71 \%$ acreditavam que vacinar os profissionais de saúde contra influenza ajudava na proteção dos pacientes, e a principal razão para se vacinarem foi proteger suas famílias e os pacientes. A maioria dos profissionais tinha conhecimento de que a vacinação contra a Influenza era realizada anualmente no país e conhecia o protocolo nacional de vacinação, porém, 
apenas $19 \%$ deles haviam recebido algum treinamento sobre Influenza e vacina contra Influenza. Além disso, somente $34 \%$ dos profissionais relataram ter recebido a vacina no último ano, sendo que os médicos foram os profissionais menos propensos a se vacinar. As variáveis relacionadas à vacinação dos profissionais foram facilidade de acesso, vacina disponível sem custo e ter recebido treinamento sobre influenza. Dos profissionais entrevistados, $94 \%$ recomendam a vacina contra influenza para pacientes soropositivos para o HIV, e as variáveis relacionadas à maior probabilidade de indicar a vacina foram a vacinação autorrelatada pelo profissional no último ano, a disponibilidade da vacina durante a consulta, ter conhecimento em relação às diretrizes de vacinação e ter recebido treinamento sobre influenza. A limitação do estudo foi ter sido realizado apenas com profissionais de saúde do serviço público e em 5 das 9 províncias sul-africanas, não sendo a amostra representativa de toda a população de profissionais de saúde ${ }^{22}$.

O terceiro estudo desta categoria avaliou nos EUA o conhecimento dos profissionais de saúde em relação ao papilomavírus humano (HPV), a prevenção do câncer cervical decorrente da vacinação e comparou os dados com um inquérito populacional realizado na mesma região. Segundo dados demonstrados pelos autores, as mulheres que vivem com HIV são três vezes mais propensas a desenvolver o câncer cervical quando comparadas com as mulheres não infectadas pelo HIV. Apesar do risco aumentado nesta população e dos benefícios da vacinação, os autores relatam que a cobertura da vacina é baixa e muitas oportunidades de vacinação são perdidas. Neste estudo, a maioria dos profissionais de saúde tinha ouvido falar na vacina contra o HPV, cerca de metade deles acreditava que era segura e eficaz, podendo proteger contra o câncer cervical, e todos os entrevistados estavam dispostos a promover a comunicação entre o profissional e o paciente sobre a vacinação contra o HPV. Um fator apontado como limitante deste estudo foi o tamanho da amostra, que por ser pequeno não possibilita generalizar os dados para toda população ${ }^{23}$.

A falta de conhecimento em relação às vacinas indicadas às PVHIV ou às questões específicas relacionadas à vacina avaliada foi identificada em três estudos (60\%) desta revisão. As atividades de educação continuada em imunização para profissionais de saúde foram apresentadas em quatro estudos $(80,0 \%)$ como importantes para aumentar a confiança dos profissionais de saúde nas vacinas e consequentemente aumentar o acesso e a cobertura vacinal nas PVHIV.

\section{DISCUSSÃO}

Esta revisão mostrou que o número de estudos que avalia o conhecimento de profissionais de saúde em relação ao processo de vacinação de PVHIV é baixo, e a maioria deles está relacionada a alguma vacina específica e não aborda todo o calendário vacinal.

Os estudos da primeira categoria apresentada destacam a falta de pesquisas que avaliem o conhecimento dos profissionais em relação ao esquema vacinal indicado para as PVHIV e reforçam a necessidade de melhor capacitar os profissionais durante a sua formação e ao longo de sua carreira, além de facilitar o acesso aos protocolos disponíveis.

A atualização do esquema vacinal do indivíduo adulto é um grande desafio, pois pouco se conhece sobre tal cobertura nesta população e acredita-se que a maioria não esteja imunizada ${ }^{24-26}$. Alguns grupos populacionais são mais suscetíveis a doenças e por isso precisam de proteção especial. Neste grupo, destacam-se as PVHIV, que caso não apresentem sinais de imunodepressão, podem ser vacinadas com todas as vacinas disponíveis no calendário de vacinação, além das especiais indicadas para eles ${ }^{4,8}$

Cabe ao profissional de saúde avaliar o estado vacinal e indicar as vacinas necessárias para iniciar ou completar o esquema de vacinação, porém, esta avaliação é um tanto complexa e exige conhecimento das vacinas disponíveis indicadas e suas contraindicações, uma vez que quanto maior for a imunodepressão, maior a possibilidade de prejuízo na resposta vacinal, além de aumentar o risco de eventos adversos $4,9,24$.

As vacinas inativadas não estão contraindicadas para pessoas imunocomprometidas, e mesmo assim observa-se na literatura baixa taxa de vacinação, além de dúvidas entre os profissionais de saúde quanto à indicação destas vacinas para imunodeprimidos ${ }^{17,27}$. O estudo realizado com PVHIV acompanhadas em um ambulatório do município de São Paulo demonstrou que somente $16,7 \%$ destas apresentavam esquema de vacinação completo para vacinas inativadas, e os autores apontaram que é necessário aumentar o conhecimento em relação às vacinas para pessoas infectadas pelo HIV em programas de educação continuada aos profissionais de saúde ${ }^{25}$.

Entretanto, para as vacinas atenuadas é necessário avaliar o risco e o benefício de realizar a vacinação levando em consideração que estes indivíduos também estão mais expostos devido ao contato frequente com os serviços de saúde. Por outro lado, os dados de segurança, eficácia e imunogenicidade das vacinas na população imunocomprometida são limitados, o que causa uma certa insegurança nos profissionais de saúde, principalmente nos médicos ao prescrever os imunobiológicos para estes pacientes ${ }^{7-9,28,29}$.

Énecessário que o profissional de saúde tenha conhecimento de marcadores importantes de imunodepressão (carga viral, contagem de CD4, medicações em uso), considerando que estas informações são de extrema importância para avaliar a indicação de vacinas para PVHIV.

Outro aspecto relevante, é a importância que o profissional de saúde desempenha na adesão à vacinação, pois melhor conhecimento sobre o assunto foi associado à maior familiaridade com o esquema de vacinação de adultos pelo profissional, à especialização deste (atenção básica/infectologia) e ao fato de a vacina ser administrada no local onde o profissional trabalha. Os pacientes tendem a confiar no profissional de saúde, por isso a indicação de vacinação por ele é importante. Para tanto, a formação do profissional precisa incluir conteúdos relacionados à vacinação de PVHIV, aplicativos que contenham 
o calendário/esquema de vacinação para este público voltados para profissionais de saúde podem auxiliar ${ }^{22}$.

A vacina contra influenza é uma vacina inativada e não tem contraindicações em pessoas soropositivas para o HIV, podendo ser administrada inclusive em indivíduos imunodeprimidos com a contagem de células T CD4 abaixo de 200 céls $/ \mathrm{mm}^{3}$, porém, estes indivíduos podem ter uma resposta prejudicada. Vacinar os profissionais de saúde e os comunicantes domiciliares contra influenza aumenta a garantia de proteção às pessoas que estejam imunossuprimidas. Além disso, os profissionais de saúde, principalmente os que fazem o acompanhamento das PVHIV, têm um papel essencial na recomendação e prescrição das vacinas para os pacientes e seus familiares ${ }^{9,26,28}$.

Nesta mesma direção, outro estudo realizado na Áustria mostrou que pacientes HIV-positivos que receberam informações sobre a vacinação do médico assistente eram mais propensos a serem vacinados contra a gripe. A recomendação direta da vacinação ao paciente foi ainda mais eficaz do que apenas informá-lo sobre a vacina. Desta forma, se os médicos informarem seus pacientes sobre a importância da vacinação e os ajudarem a superar certos fatores dificultadores, como o medo de efeitos colaterais, pode aumentar efetivamente a taxa de vacinação ${ }^{27}$.

Profissionais de saúde bem capacitados têm maior propensão a receber e a indicar a vacina, porém, não podemos deixar de destacar que atingir a cobertura vacinal depende de múltiplos fatores além da recomendação do profissional ${ }^{22}$.

Uma das formas de melhorar a cobertura vacinal em PVHIV é incorporar salas de vacinação nos ambulatórios onde eles fazem seguimento ${ }^{25}$. Em um estudo realizado em Fortaleza/ CE 4,7\% dos indivíduos com HIV informaram não ter recursos financeiros para comparecer a um serviço de vacinação. Os autores ressaltam a importância da equipe multiprofissional no processo de educação em saúde para melhoria da cobertura vacinal neste público e ressalta o papel dos profissionais de sala de vacinas para garantir que os pacientes retornem para concluir o esquema de vacinação 5 .

Em um trabalho desenvolvido no Espírito Santo onde foi avaliada a cobertura vacinal em indivíduos com HIV, a melhor cobertura foi para a vacina contra difteria e tétano $(59,79 \%)$, disponível nas salas de vacinação para todos os indivíduos, e as mais baixas coberturas foram para as vacinas com indicação especial pelos Centros de Referência em Imunobiológicos Especiais (CRIEs), vacina contra Hepatite A (6,8\%) e Meningocóccica C $(6,0 \%)$. Estes dados demonstram que mesmo para as vacinas inativadas, sem contraindicação para PVHIV, a cobertura vacinal é muito baixa ${ }^{26}$.

Uma das vacinas com indicação especial para PVHIV é a vacina contra o herpes zoster. Em um dos estudos desta revisão21, os autores ressaltam que esta é a infecção oportunista mais comum em adultos infectados pelo HIV e que estes pacientes têm maior propensão a casos graves, além da maior taxa de recorrência. Desde 2008, o uso da vacina foi padronizado na população imunocomprometida pelo Centro de Controle e Prevenção de Doenças (CDC) dos EUA e pelo Comitê Consultivo sobre Práticas de Imunização (ACIP). Apesar disso, os participantes do estudo não se sentiam seguros para indicar a vacina aos seus pacientes. Os profissionais informaram como barreira para a vacinação a falta de estudos que garantam a sua eficácia neste público, o que gera também preocupações com risco de disseminação, além de problemas para reembolso do seguro saúde. Os autores reforçam a importância da capacitação dos profissionais em relação às indicações, segurança e eficácia da vacina e a necessidade de conhecer melhor as causas da falta de conhecimento a este respeito.

No Brasil, a vacina contra herpes zoster é uma vacina de vírus vivo atenuado, recomendada para PVHIV com idade superior a 50 anos, sem sinais e sintomas de imunodepressão, porém, ela não é oferecida pelo Programa Nacional de Imunizações (PNI) e por isso está disponível apenas nas clínicas de vacinação da rede privada, o que pode levar a uma baixa cobertura vacinal contra as doenças causadas pelo herpes zoster ${ }^{28,30}$.

A preocupação com a segurança e eficácia das vacinas entre os imunodeprimidos, principalmente para as que foram introduzidas nos esquemas de vacinação mais recentemente, como a vacina contra o $\mathrm{HPV}^{23}$, é reforçada por outros autores, uma vez que pode colaborar para que muitas oportunidades de vacinação sejam perdidas. É importante que a equipe multiprofissional esteja atualizada quanto às diretrizes da vacinação, que constantemente sofrem atualizações, para que tenham segurança ao orientar e indicar a vacinação. Em um estudo desenvolvido no Brasil junto a PVHIV em atendimento ambulatorial, $63,5 \%$ dos indivíduos não foram orientados quanto ao número de doses e esquema de vacinação e $16,5 \%$ desconheciam os benefícios da mesma ${ }^{5}$.

Por fim, destaca-se que o baixo conhecimento pode estar associado com a hesitação vacinal das PVHIV que se constitui numa barreira adicional à vacinação. Estudos mostram que baixas taxas de vacinação incluem o medo dos efeitos colaterais, falta de preocupação com a prevenção, preocupação de que a vacina possa piorar o curso da infecção pelo HIV, e crença de que a vacinação falharia devido a um sistema imunológico comprometido ${ }^{27,31}$. Assim, as práticas educativas em saúde devem ser implementadas tanto para os profissionais quanto para os pacientes, para melhorar a adesão à vacinação e, consequentemente, a prevenção de doenças.

\section{CONCLUSÃO E IMPLICAÇÕES PARA A PRÁTICA}

Poucos foram os estudos identificados que abordam a temática do conhecimento dos profissionais em relação à vacinação de PVHIV e nenhum dos estudos identificados foi realizado no Brasil. O número restrito de publicações foi um fator limitante no estudo, uma vez que dificulta a análise de outros elementos que possam ser importantes no conhecimento dos profissionais de saúde em relação ao processo de vacinação das PVHIV.

A indicação de vacinas para pessoas soropositivas para o HIV exige conhecimento específico e esta avaliação não pode ser realizada apenas pelas equipes das Salas de Vacinas, evidenciando-se a necessidade de participação das equipes dos serviços de atendimento especializado e da atenção primária 
à saúde, sendo, portanto, uma responsabilidade da equipe multiprofissional.

A falta de conhecimento do profissional sobre as recomendações para a imunização das PVHIV colabora para a perda de oportunidades de vacinação e constitui em barreira para a vacinação de rotina em muitos ambientes clínicos, cenário comum atualmente na vacinação contra a Covid-19. Apesar de vários serviços brasileiros que realizam o acompanhamento clínicoambulatorial das PVHIV contarem com salas de vacinação, sua conveniência é minimizada quando os profissionais responsáveis por tal tarefa, atravessados pela insegurança, optam em não realizar o esquema de vacinação completo das PVHIV que buscam pelo atendimento desprovidos de um encaminhamento de um profissional de saúde informando o seu estado imunológico bem como as vacinas que devem receber.

O conhecimento em relação às vacinas indicadas às PVHIV e suas contraindicações deve ser compartilhado pelos profissionais de saúde em todas as esferas das redes atenção à saúde, tanto na atenção primária quanto no serviço especializado, além de envolver profissionais de saúde de diversas formações. A cobertura vacinal adequada das PVHIV contribui de forma significativa para diminuir a morbidade e mortalidade decorrentes das doenças imunopreveníveis entre as PVHIV. Para tanto, a educação destes profissionais deve ser permanente e contínua desde a sua formação. $O$ enfermeiro e a equipe de enfermagem desempenham papel de destaque no que se refere à imunização. Assim, recomendam-se estudos futuros que avaliem o conhecimento sobre a vacinação de PVHIV entre a equipe de enfermagem.

\section{CONTRIBUIÇÕES DOS AUTORES}

Desenho do estudo de revisão. Larissa Gerin. Marcela Antonini. Elucir Gir. Renata Karina Reis.

Aquisição dos textos. Larissa Gerin. Marcela Antonini. Karen da Silva Santos. Elucir Gir. Renata Karina Reis.

Análise de dados. Larissa Gerin. Marcela Antonini. Karen da Silva Santos. Elucir Gir. Renata Karina Reis.

Interpretação dos resultados. Larissa Gerin. Marcela Antonini. Karen da Silva Santos. Elucir Gir. Renata Karina Reis.

Redação e revisão crítica do manuscrito. Larissa Gerin. Marcela Antonini. Karen da Silva Santos. Elucir Gir. Renata Karina Reis.

Aprovação da versão final do artigo. Larissa Gerin. Marcela Antonini. Karen da Silva Santos. Elucir Gir. Renata Karina Reis.

Responsabilidade por todos os aspectos do conteúdo e a integridade do artigo publicado. Larissa Gerin. Marcela Antonini. Karen da Silva Santos. Elucir Gir. Renata Karina Reis.

\section{EDITOR ASSOCIADO}

Rodrigo Nogueira da Silva (1)

\section{EDITOR CIENTÍFICO}

Ivone Evangelista Cabral (1)

\section{REFERÊNCIAS}

1. Unaids. Estatísticas, 2021 [Internet]. Brasília: Unaids; 2021 [cited 2021 Jan 22]. Available from: https://unaids.org.br/estatisticas/?gclid=EAlal QobChMlql3g7Mav7gIVjISRCh125Q-2EAAYASABEgKp1_D_BwE.

2. Ministério da Saúde (BR). Boletim Epidemiológico HIV/aids 2020 [Internet]. Brasília: Ministério da Saúde; 2020 [cited 2021 Jan 22]. Available from: http://www.aids.gov.br/pt-br/pub/2020/boletim-epidemiologicohivaids-2020.

3. Melo EA, Maksud I, Agostini R. Cuidado, HIV/Aids e atenção primária no Brasil: desafio para a atenção no Sistema Único de Saúde? Rev Panam Salud Publica. 2018 ct;42:e151. http://dx.doi.org/10.26633/ RPSP.2018.151. PMid:31093179.

4. Ministério da Saúde (BR). Protocolo clínico e diretrizes terapêuticas para manejo da infecção pelo HIV em adultos. Brasília: Ministério da Saúde; 2018.

5. Cunha GH, Galvão MTG, Medeiros CM, Rocha RP, Lima MAC, Fechine FV.Vaccination status of people living with HIV/AIDS in outpatient care in Fortaleza, Ceará, Brazil. Braz J Infect Dis. 2016 Sep/Oct;20(5):48793. http://dx.doi.org/10.1016/j.bjid.2016.07.006. PMid:27542868.

6. Johnson TM, Klepser DG, Bares SH, Scarsi KK. Predictors of vaccination rates in people living with HIV followed at a specialty care clinic. Hum Vaccin Immunother. 2021 Sep;17(3):791-6. http://dx.doi.org/10.1080/ 21645515.2020.1802163. PMid:32881642.

7. Ministério da Saúde (BR). Manual dos Centros de Referência para Imunobiológicos Especiais. Brasília: Ministério da Saúde; 2019

8. Chaer FE, Sahly HM. Vaccination in the adult patient infected with HIV: a review of vaccine efficacy and immunogenicity. Am J Med. 2019 Apr;132(4):437-46. http://dx.doi.org/10.1016/j.amjmed.2018.12.011. PMid:30611828.

9. Rubin LG, Levin MJ, Ljungman P, Davies G, Avery R, Tomblyn $M$ et al. 2013 IDSA Clinical Practice Guideline for Vaccination of the Immunocompromised Host. Clin Infect Dis. 2014 Feb;58(3):e44-100. http://dx.doi.org/10.1093/cid/cit684. PMid:24311479.

10. Soares CB, Hoga LAK, Peduzzi M, Sangaleti C, Yonekura T, Silva DRAD. Revisão integrativa: conceitos e métodos utilizados na enfermagem. Rev Esc Enferm USP. 2014 Apr;48(2):335-45. http://dx.doi.org/10.1590/ S0080-6234201400002000020. PMid:24918895.

11. Souza MT, Silva MD, Carvalho R. Revisão integrativa: o que é e como fazer. Einstein. 2010 Jan/Mar:8(1):102-6. http://dx.doi.org/10.1590/ s1679-45082010rw1134. PMid:26761761.

12. Whittemore R, Knafl K. The integrative review: updated methodology J Adv Nurs. 2005 Dec;52(5):546-53. http://dx.doi.org/10.1111/j.13652648.2005.03621.x. PMid:16268861.

13. Cardoso V, Trevisan I, Cicotella DA, Waterkemper R. Revisão sistemática de métodos mistos: método de pesquisa para a incorporação de evidências na enfermagem. Texto Contexto Enferm. 2019 Apr;28:e20170279. http:// dx.doi.org/10.1590/1980-265x-tce-2017-0279.

14. Ouzzani M, Hammady H, Fedorowicz Z, Elmagarmid A. Rayyan - a web and mobile app for systematic reviews. Syst Rev. 2016 Dec;5(1):210. http://dx.doi.org/10.1186/s13643-016-0384-4. PMid:27919275.

15. Melnyk BM, Fineout-Overholt E. Evidence-based practice in nursing and healthcare: a guide to best practice. Philadelphia: Lippincot Williams \& Wilkins; 2005

16. Sá GGM, Silva FL, Santos AMR, Nolêto JS, Gouveia MTO, Nogueira LT. Tecnologias desenvolvidas para a educação em saúde de idosos na comunidade: revisão integrativa da literatura. Rev Lat Am Enfermagem. 2019 Oct;27:e3186. http://dx.doi.org/10.1590/1518-8345.3171.3186. PMid:31618386.

17. Moer D, Loberati A, Tetzlaff J, Altman DG. Preferred reporting items for systematic reviews and meta-analysis: the PRISMA statement. PLoS Med. 2009 Jul;6(7):339. http://dx.doi.org/10.1136/bmj.b2535.

18. Vaismoradi $\mathrm{M}$, Turunen $\mathrm{H}$, Bondas $\mathrm{T}$. Content analysis and thematic analysis: implications for conducting a qualitative descriptive study. Nurs Health Sci. 2013 Sep;15(3):398-405. http://dx.doi.org/10.1111/ nhs.12048. PMid:23480423.

19. Allen JE, Patel D. Enquiries to the United Kingdom National Travel Advice Line by healthcare professionals regarding immunocompromised 
travellers. JTravel Med. 2016 Mar;23(3):taw016. http://dx.doi.org/10.1093/ jtm/taw016. PMid:27021497.

20. Blackwell CW. Knowledge of Vaccination Needs of HIV-infected men who have sex with men in a national sample of "gay friendly" health care providers. Public Health Nurs. 2016 Sep;33(5):403-11. http://dx.doi org/10.1111/phn.12250. PMid:26847956.

21. Aziz M, Kessler H, Huhn G. Providers' lack of knowledge about herpes zoster in HIV-infected patients is among barriers to herpes zoster vaccination. Int J STD AIDS. 2013 Jul;24(6):433-9. http://dx.doi. org/10.1177/0956462412472461. PMid:23970744.

22. Wigfall LT, Bynum SA, Brandt HM, Sebastian N, Ory MG. HPV-related cancer prevention and control programs at community-based hiv/ aids service organizations: implications for future engagement. Front Oncol. 2018 Oct;8:422. http://dx.doi.org/10.3389/fonc.2018.00422. PMid:30416981.

23. Center for Disease Control and Prevention. Adult immunization: knowledge, attitudes, and practices -- DeKalb and Fulton Counties, Georgia, 1988. MMWR Morb Mortal Wkly Rep [Internet]. 1988; [cited 2021 Jan 22];37(43):657-61. Available from: https://www.cdc.gov/mmwr/ preview/mmwrhtml/00021583.htm

24. Ho YL, Enohata T, Lopes MH, Santos SS. Vaccination in Brazilian HIV-infected adults: a cross-sectional study. AIDS Patient Care STDS. 2008 Jan;22(1):65-70. http://dx.doi.org/10.1089/apc.2007.0059. PMid:18095834.

25. Pinto No LFSP, Vieira JV, Ronchi NR. Vaccination coverage in a cohort of HIV - infected patients receiving a care at an AIDS outpatient clinic in Espírito Santo, Brazil. Braz J Infect Dis. 2017 Sep/Oct;21(5):515-9. http://dx.doi.org/10.1016/j.bjid.2017.03.021. PMid:28579170.

26. Harrison N, Poeppl W, Herkner RH, Tillhof KD, Grabmeier-Pfistershammer $\mathrm{K}$, Rieger $\mathrm{A}$ et al. Predictors for and coverage of influenza vaccination among HIV-positive patients: a cross-sectional survey. HIV Med. 2017 Dec;18(7):500-6. http://dx.doi.org/10.1111/hiv.12483. PMid:28035738.

27. Sociedade Brasileira de Imunizações (SBIm). Calendário de vacinação SBIm pacientes especiais 2020-2021. São Paulo: SBIm; 2020.

28. Nolte F, Pacchiotti A, Castellano V, Lamy P, Gentile A. Reticencia a la vacunación: abordaje de su complejidad. Rev Hosp Ninos "Alejandro Mann". 2016;58(261):16-22.

29. Duque J, Gaga S, Clark D, Muller M, Kuwane B, Cohen C et al. Knowledge attitudes and practices of South African healthcare workers regarding the prevention and treatment of influenza among HIV-infected individuals. PLoS One.2017 Mar;12(3):e0173983. http://dx.doi.org/10.1371/journal. pone.0173983. PMid:28301593.

30. Erlandson KM, Streifel A, Novin AR, Hawkins KL, Foster C, Langness $J$ et al. Low rates of vaccination for herpes zoster in older people living with HIV. AIDS Res Hum Retroviruses. 2018 Jul;34(7):603-6. http:/ dx.doi.org/10.1089/aid.2017.0315. PMid:29661022.

31. Moyroud L, Hustache S, Goirand L, Hauzanneau M, Epaulard O. Negative perceptions of hepatitis $B$ vaccination among attendees of an urban free testing center for sexually transmitted infections in France. Hum Vaccin Immunother. 2017 May;13(5):998-1004. http://dx.doi.org /10.1080/21645515.2016.1264549. PMid:27937074. 